\title{
Messen und Prüfen in der Klebtechnik
}

Die durchgängige Qualitätssicherung ist für den wirtschaftlichen Erfolg der Klebtechnik bekanntlich unerlässlich. Einen wichtigen Part übernehmen in diesem Zusammenhang die verschiedenen auf dem Markt verfügbaren Mess- und Prüfsysteme.

$\mathrm{D}$ ie Klebtechnik gilt in Verbindung mit neuen Werkstoffen als unverzichtbar und hat schon lange ihre Serientauglichkeit unter Beweis gestellt. Wichtige Voraussetzung für den wirtschaftlichen Einsatz der Klebtechnik ist allerdings eine durchgängige Qualitätssicherung. Wie bei allen anderen Fügeverfahren bedarf es hier eines Qualitätskonzeptes, das bei der Produktentwicklung beginnt und sich bis zur fertigen Klebverbindung erstreckt. Die Auswahl des jeweiligen Verfahrens für die Prüfung oder Messung richtet sich nach den zu ermittelnden Parametern und den jeweiligen Umständen, unter denen geprüft werden soll. Es stellt sich dabei die Frage, ob es sich um qualitätssichernde Kontrollen während des Produktionsprozesses oder um Prüfungen im Labor handelt und ob physikalische GröBen oder ein Verhalten bei Beanspruchung zu ermitteln sind. Um Festigkeitswerte $\mathrm{zu}$ messen, werden Klebungen verschiedenen Beanspruchungsarten unterworfen und zerstörend geprüft, wobei zwischen statischen und dynamischen Verfahren unterschieden wird. Mit zerstörungsfreien Prüfverfahren lassen sich u. a. Klebschichten auf Fehlstellen, der Klebstoffauftrag nach Lage und Menge, die Benetzung des Fügeteils oder der Härtungsverlauf überwachen.

Wichtig sind in diesem Zusammenhang auch die Rheometer/Viskosime- ter, durch deren Einsatz sich zum Beispiel die Benetzungsfähigkeit von Klebstoffen auf Fügeoberflächen optimieren lässt.

Mit der folgenden tabellarischen Auflistung wollen wir potenziellen Anwendern der Klebtechnik einen Überblick über die Lieferanten zerstörungsfreier und zerstörender Systeme für den klebtechnischen Einsatz bieten. Das Angebotsspektrum erstreckt sich von Systemen zur Oberflächencharakterisierung über die Rheometrie bis hin zur Bewitterungsprüfung.

\section{Wenn Ihr}

\section{Unternehmen fehlt}

Diese Marktübersicht wird jährlich aktualisiert und um neue Firmen mit den entsprechenden Angeboten ergänzt. Unternehmen, die zukünftig ebenfalls in der Marktübersicht mit ihren Systemen und Geräten zum Messen und Prüfen in der Klebtechnik genannt werden möchten, können einen Eintragungsbogen anfordern bei: adhaesion@springer.com. 